\title{
Fluoroscopically-Guided Pulsed Radiofrequency Neurotomy Technique for the Treatment of Genitofemoral Neuralgia
}

\section{TO The Editor}

A 48-year-old woman presented to our clinic for evaluation of persistent, burning, left-sided groin pain that occurred after placement of a bone-anchored midurethral sling 8 years prior. The pain persisted despite urethrolysis, tension-free transvaginal tape placement, and eventual sling and bone anchor removal. Multiple pharmacologic agents failed to control her symptoms.

She presented to our clinic about 6 months after sling and bone anchor removal. Ultrasound-guided genitofemoral (GF) nerve blocks repeatedly gave her over $50 \%$ relief for 3 weeks at a time. Due to her favorable response to GF nerve blockade she was deemed an optimal candidate for pulsed radiofrequency neurotomy.

For the procedure, the patient was positioned supine. Under fluoroscopy, an anterior-posterior view of the pelvis containing the anterior superior iliac spine (ASIS) and the left pubic tubercle was obtained. An initial puncture site was marked approximately $3 \mathrm{~cm}$ superolateral from the left pubic tubercle along a line extending from the left pubic tubercle to the ASIS. After cleaning and anesthetizing the skin, a 20 gauge, $10 \mathrm{~mm}$ active-tip, radiofrequency cannula (Baylis Medical Com- pany) was inserted at a 45 degree angle with the tip directed from lateral to medial. Once the superior pubic ramus was contacted, the cannula was withdrawn and repositioned at a slightly more oblique angle. This was repeated until the active node of the cannula was approximately $1 \mathrm{~cm}$ lateral to the left pubic tubercle at an angle parallel to the genitofemoral nerve. Fluoroscopy was used to ensure position over bone for the entirety of the procedure (Fig. 1). Contrast confirmed the absence of vascular flow. Sensory and motor stimulation was tested at $50 \mathrm{MHz}$ and $2 \mathrm{MHz}$, respectively. On sensory testing, the patient reported a reproduction of pain in her typical distribution. After anesthetizing with $1 \mathrm{~mL}$ of $1 \%$ lidocaine, the nerve was ablated on a pulsed setting with $2 \mathrm{~Hz}$ frequency and $20 \mathrm{~ms}$ pulse duration. The peak temperature was 42 degrees and the voltage ranged between 50 and 60 volts. The nerve was ablated twice for 120 seconds each time. After the ablation, $1 \mathrm{~mL}$ of $0.25 \%$ bupivacaine and $1 \mathrm{~mL}$ of 40 $\mathrm{mg} / \mathrm{mL}$ of triamcinolone was injected.

Prior to the neurotomy the patient reported her pain as 8 out of 10 on a numerical pain rating scale.

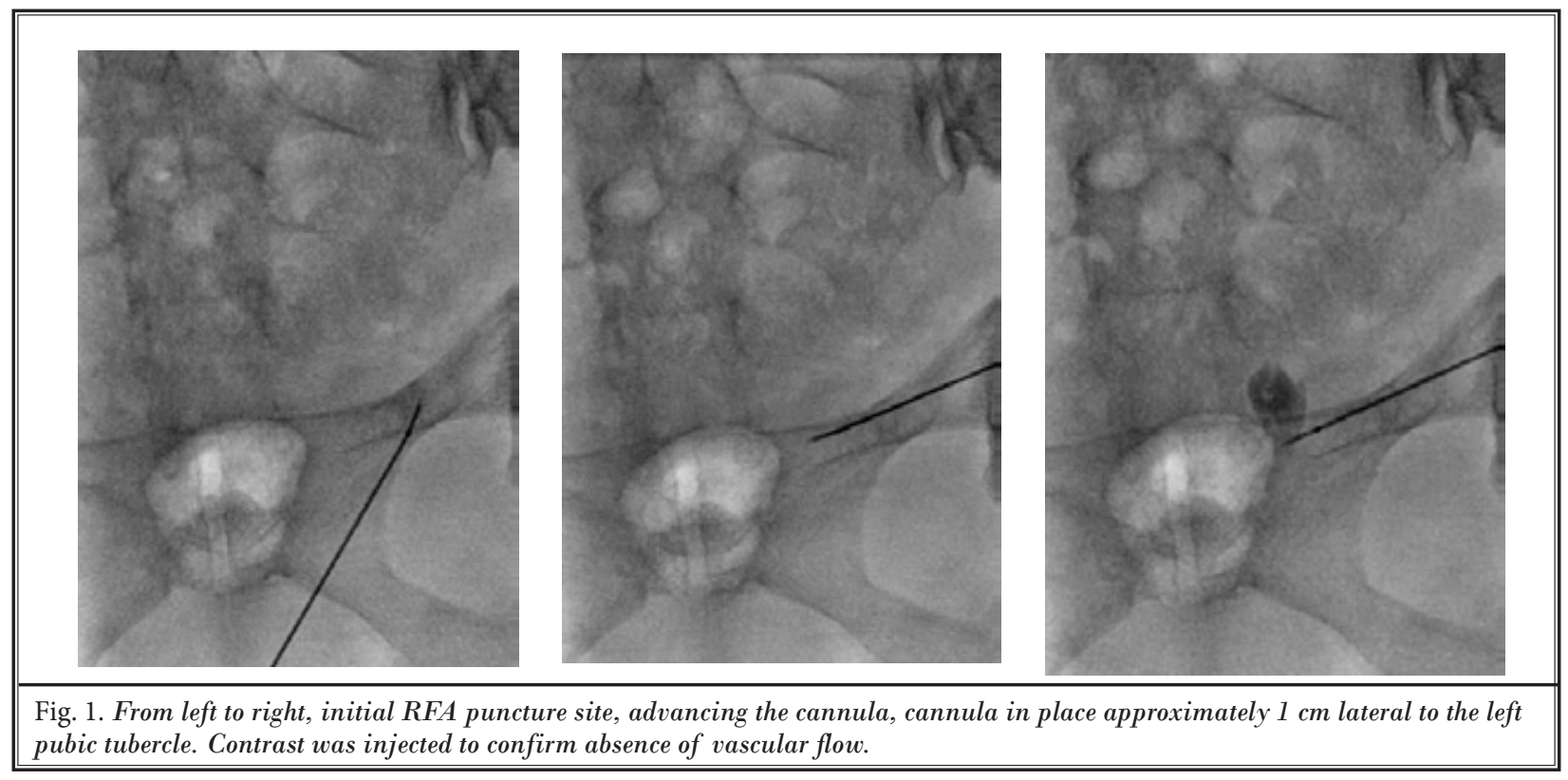




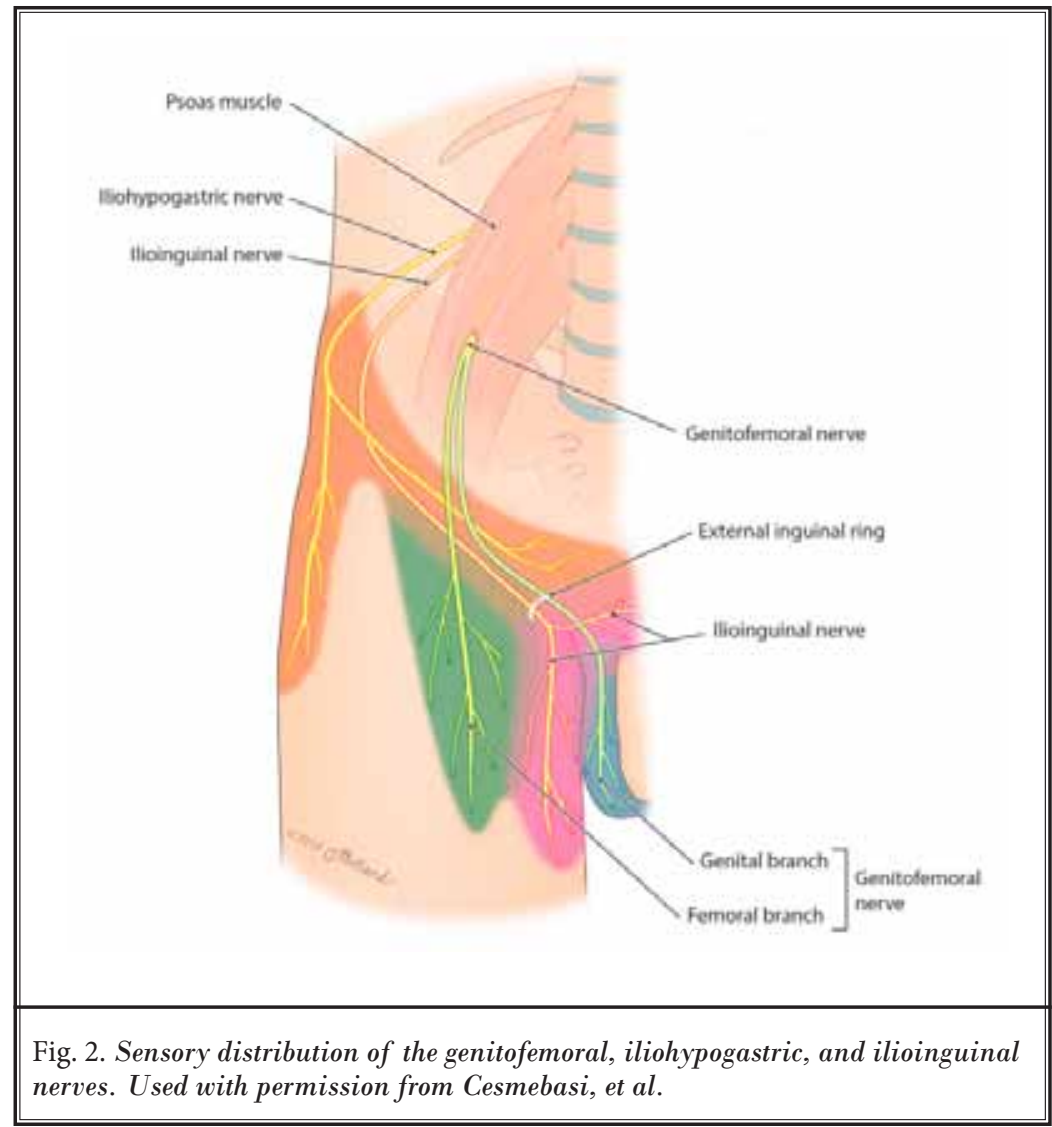

Immediately post-neurotomy, the patient reported a $100 \%$ relief of her pain. At follow up 1.5 months post-procedure she reported sustained $95 \%$ pain relief.

Genitofemoral neuralgia (GFN) was first described by McGee in 1942 (1). GFN causes pain in the groin and medial thigh. The etiology is thought to be from nerve entrapment or iatrogenic injury. GFN has been noted after various inguinal and abdominal surgeries (2). The exact prevalence of GFN is unknown.

The genitofemoral nerve originates from the L1 and L2 ventral spinal nerve roots, travels within the psoas muscle until about the L3/ L4 level where the nerve pierces the anterior surface of the psoas. It then divides into the genital and femoral branches. The genital branch continues along the psoas, enters the deep inguinal ring and, in men, supplies the spermatic cord, sensory innervation to the scrotum, and motor innervation to the cremaster muscle. In women, the genital branch runs alongside the round ligament and provides innervation to the mons pubis and labia majora. The femoral branch travels lateral to the femoral artery and posterior to the inguinal ligament, piercing the sartorius to innervate the skin of the anteromedial thigh around the femoral triangle (Fig. 2).

A variety of interventional techniques to treat GFN have been described in the literature including CT-guided transpsoas injection $(3,4)$, ultrasound-guided blockade of the genitofemoral nerve at the inguinal canal (5), ultrasound-guided cryoablation (6), pulsed radiofrequency neurotomy (PFN) with anatomic guidance (7), and ultrasound-guided PFN (8). Only one study used fluoroscopy to guide a tack injection to treat GFN following inguinal herniorrhaphy (9).

To our knowledge, the case described above is the first to describe a fluoroscopically-guided pulsed radiofrequency neurotomy technique for the treatment of GFN. While ultrasound and CT are appropriate methods for guidance, the availability of these modalities may vary from clinic to clinic. In addition, clinicians' expertise with ultrasound and CT-guidance may also vary significantly. For those not as comfortable with CT or ultrasound guidance, fluoroscopic guidance may be a viable alternative.

Vincent F. Miccio, Jr, MD

Fellow

Columbia University Medical Center

Department of Anesthesiology

Division of Pain Medicine

E-mail: vincent.miccio@gmail.com

Rohit Navlani, DO

PGY-1

Dept. of Physical Medicine

\& Rehabilitation

University of Pittsburgh

Pittsburgh, PA

E-mail: navlani.rohit@gmail.com

Jaclyn H. Bonder, MD

Dept. of Rehabilitation

NewYork-Presbyterian Hospital

Weill Cornell Medical Center

E-mail: jab9155@med.cornell.edu

Jaspal Ricky Singh, MD

Dept. of Rehabilitation

NewYork-Presbyterian Hospital

Weill Cornell Medical Center

E-mail: jrs9012@med.cornell.edu 


\section{References}

1. Magee RK. Genitofemoral Causalgia: (A New Syndrome). Can Med Assoc J 1942; 46:326-329.

2. Cesmebasi A, Yadav A, Gielecki J, Tubbs RS, Loukas M. Genitofemoral neuralgia: a review. Clin Anat 2015; 28:128-135.

3. Parris D, Fischbein N, Mackey S, Carroll I. A novel CT-guided transpsoas approach to diagnostic genitofemoral nerve block and ablation. Pain Med 2010;11:785-789.

4. Hackworth RJ, Nagel EJ, Slotto JG. Computed tomographic-guided genitofemoral nerve block: A simple anterior ap- proach. J Comput Assist Tomogr 2015; 39:295-297.

5. Shanthanna H. Successful treatment of genitofemoral neuralgia using ultrasound guided injection: a case report and short review of literature. Case Rep Anesthesiol 2014; 2014:371703.

6. Campos NA, Chiles JH, Plunkett AR. UItrasound-guided cryoablation of genitofemoral nerve for chronic inguinal pain. Pain Physician 2009; 12:997-1000.

7. Cohen SP, Foster A. Pulsed radiofre- quency as a treatment for groin pain and orchialgia. Urology 2003; 61:645.

8. Terkawi AS, Romdhane K. Ultrasoundguided pulsed radiofrequency ablation of the genital branch of the genitofemoral nerve for treatment of intractable orchalgia. Saudi] Anaesth. 2014; 8:294.

9. Rho, R. H., Lamer, T. J. and Fulmer, J. T. Treatment of Genitofemoral neuralgia after laparoscopic inguinal herniorrhaphy with fluoroscopically guided tack Injection. Pain Medicine 2001; 2:230-233. 